\title{
Zeckendorf representations with at most two terms to $x$-coordinates of Pell equations
}

\author{
Carlos Alexis Gómez Ruiz \\ Departamento de Matemáticas, Universidad del Valle, \\ Calle 13 No 100-00, Cali, Colombia \\ carlos.a.gomez@correounivalle.edu.co \\ FLORIAN LUCA \\ School of Mathematics, University of the Witwatersrand, \\ Private Bag X3, Wits 2050, South Africa \\ Max Planck Institute for Mathematics, \\ Vivatgasse 7, 53111 Bonn, Germany \\ Department of Mathematics, Faculty of Sciences, \\ University of Ostrava, 30 Dubna 22, 70103 Ostrava 1, Czech Republic \\ florian.luca@wits.ac.za
}

November 15, 2018

\begin{abstract}
In this paper, we find all positive squarefree integers $d$ such that the Pell equation $X^{2}-d Y^{2}=$ \pm 1 has at least two positive integer solutions $(X, Y)$ and $\left(X^{\prime}, Y^{\prime}\right)$ such that both $X$ and $X^{\prime}$ have Zeckendorf representations with at most two terms.

This paper has been accepted for publication in SCIENCE CHINA Mathematics.
\end{abstract}

Key words and phrases. Pell equation, Fibonacci numbers, Lower bounds for linear forms in logarithms, Reduction method.

2010 Mathematics Subject Classification. 11B39, 11J86.

\section{Introduction}

For a positive squarefree integer $d$ and the Pell equation

$$
X^{2}-d Y^{2}= \pm 1, \quad \text { where } \quad X, Y \in \mathbb{Z}^{+}
$$

it is well-known that all its solutions $(X, Y)$ have the form

$$
X+Y \sqrt{d}=X_{k}+Y_{k} \sqrt{d}=\left(X_{1}+Y_{1} \sqrt{d}\right)^{k}
$$


for some $k \in \mathbb{Z}^{+}$, where $\left(X_{1}, Y_{1}\right)$ be the smallest positive integer solution of (11). The sequence $\left\{X_{k}\right\}_{k \geq 1}$ is a binary recurrent sequence. In fact, the formula

$$
X_{k}=\frac{\left(X_{1}+\sqrt{d} Y_{1}\right)^{k}+\left(X_{1}-\sqrt{d} Y_{1}\right)^{k}}{2}
$$

holds for all positive integers $k$.

Recently there was a spur of activity around investigating for which $d$, there are members of sequence $\left\{X_{k}\right\}_{k \geq 1}$ which belong to some interesting sequences of positive integers. Maybe the first result of this kind is due to Ljunggren [7] who showed that if (1) has a solution with -1 on the right-hand side, then there is at most one odd $k$ such that $X_{k}$ is a square. In [2], it is shown that if all solutions of (11) have the sign +1 on the right-hand side, then $X_{k}$ is a square only when $k \in\{1,2\}$, with both $X_{1}$ and $X_{2}$ being squares occurring only for $d=1785$. When only solutions with the sign +1 in the right-hand side are considered, in [3] it is shown that $X_{k}$ is a repdigit in base 10 for at most one $k$, except when $d=2$, for which both $X_{1}=3$ and $X_{3}=99$ are repdigits, and when $d=3$ for which both $X_{1}=2$ and $X_{2}=7$ are repdigits. More generally, in [5] it is shown that if $b \geq 2$ is any integer, then, under the same assumption that only solutions with the sign +1 on the right-hand side are considered, there are only finitely many $d$ 's such that $X_{k}$ is a base $b$-repdigit for at least two values of $k$. All such $d$ are bounded by $\exp \left((10 b)^{10^{5}}\right)$. In $[9$, it is shown that $X_{k}$ is a Fibonacci number for at most one $k$, except for $d=2$ when both $X_{1}=1$ and $X_{2}=3$ are Fibonacci numbers.

We recall that the Fibonacci sequence $\left\{F_{k}\right\}_{k \geq 0}$ and its companion Lucas sequence $\left\{L_{k}\right\}_{k \geq 0}$ are given by $F_{0}=0, F_{1}=1, L_{0}=2, L_{1}=1$ and for both, each term afterwards is the sum of the preceding two terms.

Letting $\alpha=(1+\sqrt{5}) / 2$ and $\beta=(1-\sqrt{5}) / 2$ be the roots of the characteristic polynomial $X^{2}-X-1$ of both the Fibonacci and Lucas sequences, the Binet formulas

$$
F_{k}=\frac{\alpha^{k}-\beta^{k}}{\sqrt{5}} \quad \text { and } \quad L_{k}=\alpha^{k}+\beta^{k}
$$

hold for all nonnegative integers $k$. Further, the inequalities

$$
\alpha^{k-2} \leq F_{k} \leq \alpha^{k-1} \quad \text { hold for all } \quad k \geq 1 .
$$

Zeckendorf's theorem (see [14]) claims that every positive integer $N$ has a unique representation as sum of non-consecutive Fibonacci numbers. That is,

$$
N=F_{k_{1}}+\cdots+F_{k_{r}}, \quad \text { where } \quad k_{i+1}-k_{i} \geq 2 \quad \text { for all } \quad i=1,2, \ldots, r-1 .
$$

We say that $N$ has Zeckendorf representation with $r$ terms.

In this paper, we look at Pell equations (10) such that $X_{\ell}$ has Zeckendorf representation with at most two terms, for at least two values of $\ell$.

We prove the following result.

Theorem 1. For each squarefree integer $d$, there is at most one positive integer $\ell$ such that $X_{\ell}$ has a Zeckendorf representation with at most two terms, except for $d \in\{2,3,5,11,30\}$.

For the exceptional values of $d$ appearing in the statement of Theorem 1, all solutions $(\ell, m, n)$ of the Diophantine equation

$$
X_{\ell}=F_{m}+F_{n}, \quad \text { with } \quad n-m \geq 2
$$


are listed in the Section 2 and 5. Note that our results also give all solutions of the problem under the more relaxed condition that $n \geq m$ (but not necessarily that $n-m \geq 2$ ). Namely, if $m=n-1$, we then take $F_{n}+F_{n-1}=F_{n+1}+F_{0}$ and when $m=n\left(\right.$ and $n \geq 2$, since $F_{1}=F_{2}$ ), then $F_{n}+F_{m}=2 F_{n}=F_{n+1}+F_{n-2}$.

The main tools used in this work are lower bounds for linear forms in logarithms á la Baker and a version of the Baker-Davenport reduction method from Diophantine approximation, in addition to elementary properties of Fibonacci numbers and solutions to Pell equations.

\section{A preliminary consideration}

First of all, we consider the case $d=5$ in equation (1). It is well-known that if $(X, Y)$ are positive integers such that

$$
X^{2}-5 Y^{2}= \pm 4, \quad \text { then } \quad(X, Y)=\left(L_{n}, F_{n}\right) \text { for some } n \geq 1 .
$$

In particular, if $X^{2}-5 Y^{2}= \pm 1$, then $(2 X)^{2}-5(2 Y)^{2}= \pm 4$, so $2 X=L_{n}$ for some integer $n$. Thus, $X=L_{n} / 2$ and since this is an integer, we have $3 \mid n$. One checks (by induction, for example), that

$$
\frac{L_{n}}{2}=F_{n}+\frac{F_{n-3}}{2} \text {. }
$$

So, assume that $L_{n} / 2=F_{a}+F_{b}$ for some $n \geq 10$ and $a \geq b$ (in particular for $a-b \geq 2$ ). If $a \leq n-2$, then

$$
F_{n}<F_{n}+\frac{F_{n-3}}{2}=\frac{L_{n}}{2}=F_{a}+F_{b} \leq 2 F_{a} \leq F_{n-2}<F_{n}
$$

a contradiction. If $a=n-1$ and $b \leq n-2$, then again

$$
F_{n}<\frac{L_{n}}{2}=F_{a}+F_{b} \leq F_{n-1}+F_{n-2}=F_{n}
$$

a contradiction, while if $a=b=n-1$, then

$$
F_{a}+F_{b}=2 F_{n-1}=F_{n}+F_{n-3}>F_{n}+\frac{F_{n-3}}{2}=\frac{L_{n}}{2},
$$

again a contradiction. Certainly, if $a \geq n+1$, then

$$
F_{a}+F_{b} \geq F_{n+1}=F_{n}+F_{n-1}>F_{n}+\frac{F_{n-3}}{2}=\frac{L_{n}}{2},
$$

again a contradiction. Having explored both possibilities $a \geq n+1$ and $a \leq n-1$ without success, we conclude that $a=n$. Hence,

$$
F_{a}+F_{b}=F_{n}+F_{b}=F_{n}+\frac{F_{n-3}}{2}=\frac{L_{n}}{2},
$$

giving $F_{b}=F_{n-3} / 2$. This is also wrong since

$$
F_{n-5}<\frac{F_{n-3}}{2}<F_{n-4}
$$

for $n \geq 10$ meaning $F_{n-3} / 2$ cannot be a Fibonacci number $F_{b}$. Thus, $n<10$, and since $3 \mid n$ we need to check $n=3,6,9$. The cases $n=3,6$ give the solutions

$$
\begin{array}{r}
F_{3}+F_{0}=2 F_{2}=2 F_{1}=F_{2}+F_{1}=2=X_{1}, \\
F_{6}+F_{1}=F_{6}+F_{2}=9=X_{2},
\end{array}
$$


while the case $n=9$ doesn't since for it we have $X_{3}=L_{9} / 2=38$ which is not a sum of two Fibonacci numbers.

From now on, we will consider the Pell equation (1) with $d \neq 5$.

\section{An inequality for $n$ and $\ell$}

Let $\left(X_{1}, Y_{1}\right)$ be the minimal solution in positive integers of the Pell equation (1) with $d \neq 5$. Taking

$$
\delta:=X_{1}+\sqrt{d} Y_{1} \quad \text { and } \quad \eta:=X_{1}-\sqrt{d} Y_{1} .
$$

We obtain that

$$
\delta \cdot \eta=X_{1}^{2}-d Y_{1}^{2}=: \epsilon, \quad \epsilon \in\{ \pm 1\}
$$

Thus, from (21), we have

$$
X_{\ell}=\frac{1}{2}\left(\delta^{\ell}+\eta^{\ell}\right) .
$$

Since $\delta \geq 1+\sqrt{2}$ and $\alpha=(1+\sqrt{5}) / 2$, it follows that the estimate

$$
\frac{\delta^{\ell}}{\alpha^{2}} \leq X_{\ell}<\delta^{\ell} \quad \text { holds for all } \quad \ell \geq 1 .
$$

Indeed, inequality on the right-hand side is taken from the fact that $|\eta|=|\delta|^{-1}$. To inequality on the left-hand side, we note that

$$
X_{\ell} \geq \frac{\delta^{\ell}-\delta^{-\ell}}{2}=\delta^{\ell}\left(\frac{1-\delta^{-2 \ell}}{2}\right) \geq \delta^{\ell}\left(\frac{1-(1+\sqrt{2})^{-2}}{2}\right)>\frac{\delta^{\ell}}{\alpha^{2}} .
$$

We assume that $\left(m_{1}, n_{1}, \ell_{1}\right)$ and $\left(m_{2}, n_{2}, \ell_{2}\right)$ are shortlists of positive integers such that

$$
F_{m_{1}}+F_{n_{1}}=X_{\ell_{1}} \quad \text { and } \quad F_{m_{2}}+F_{n_{2}}=X_{\ell_{2}}
$$

with $1 \leq \ell_{1}<\ell_{2}$. We also assume that $n_{i}-m_{i} \geq 2$ for $i=1,2$. By the main result in [9], we may assume that not both $m_{1}$ and $m_{2}$ are zero, although this condition will not be used. Thus, $n_{i} \geq 2$ for $i=1,2$.

We will start from the assumption that $n \geq 3$. Setting $(m, n, \ell):=\left(m_{i}, n_{i}, \ell_{i}\right)$, for $i \in\{1,2\}$ and using inequalities (4) and (7), we get from (8) that

$$
\alpha^{n-2} \leq \alpha^{m-2}+\alpha^{n-2} \leq F_{m}+F_{n}=X_{\ell} \leq \delta^{\ell}
$$

and

$$
\frac{\delta^{\ell}}{\alpha^{2}} \leq X_{\ell}=F_{m}+F_{n} \leq \alpha^{m-1}+\alpha^{n-1} \leq \alpha^{n-1}\left(1+\alpha^{-2}\right) .
$$

The above inequalities give

$$
(n-2) \log \alpha<\ell \log \delta \leq(n+1) \log \alpha+\log \left(1+\alpha^{-2}\right) .
$$

Dividing across by $\log \alpha$ and setting $c_{1}:=1 / \log \alpha$, we deduce that

$$
-2<c_{1} \ell \log \delta-n<1+\frac{\log \left(1+\alpha^{-2}\right)}{\log \alpha},
$$


and since $\alpha^{2}=\alpha+1>2$, we get

$$
\left|n-c_{1} \ell \log \delta\right| \leq 2
$$

Furthermore, $\ell<n$, for if not, we would then get

$$
\delta^{n} \leq \delta^{\ell} \leq \alpha^{n+1}\left(1+\alpha^{-2}\right), \quad \text { implying } \quad\left(\frac{\delta}{\alpha}\right)^{n} \leq \alpha+\alpha^{-1}
$$

which is false since $\delta \geq 1+\sqrt{2}, \alpha=(1+\sqrt{5}) / 2$ and $n \geq 3$.

Besides, given that $\ell_{1}<\ell_{2}$, we have by (8) and (4) that

$$
\alpha^{n_{1}-2} \leq F_{n_{1}} \leq F_{m_{1}}+F_{n_{1}}=X_{\ell_{1}}<X_{\ell_{2}}=F_{m_{2}}+F_{n_{2}}<\alpha^{n_{2}-1}\left(1+\alpha^{-2}\right) .
$$

Thus,

$$
n_{1} \leq n_{2}+2
$$

Using identities (6) and (3) in the Diophantine equations (8), we get

$$
\frac{\alpha^{m}+\alpha^{n}}{\sqrt{5}}-\frac{1}{2} \delta^{\ell}=\frac{1}{2} \eta^{\ell}+\frac{\beta^{m}+\beta^{n}}{\sqrt{5}} .
$$

Thus, dividing both sides of the above equality by $\left(\alpha^{n}+\alpha^{m}\right) / \sqrt{5}$ and taking absolute value, we get

$$
\left|\delta^{\ell}(\sqrt{5} / 2) \alpha^{-n}\left(1+\alpha^{m-n}\right)^{-1}-1\right|<\frac{3.6}{\alpha^{n}},
$$

where we have used the facts that $|\eta|=|\delta|^{-1},|\beta|=\alpha^{-1}, \ell \geq 1$ and $m \geq 0$.

Put

$$
\Lambda_{1}:=\delta^{\ell}(\sqrt{5} / 2) \alpha^{-n}\left(1+\alpha^{m-n}\right)^{-1}-1,
$$

and

$$
\Gamma_{1}:=\ell \log \delta+\log (\sqrt{5} / 2)-n \log \alpha-\log \left(1+\alpha^{m-n}\right) .
$$

Since $\left|e^{\Gamma_{1}}-1\right|=\left|\Lambda_{1}\right|<3.6 / \alpha^{n}<0.84$ for $n \geq 3$, it follows that $e^{\left|\Gamma_{1}\right|}<6.25$ and so

$$
\left|\Gamma_{1}\right|<e^{\left|\Gamma_{1}\right|}\left|e^{\Gamma_{1}}-1\right|<\frac{23}{\alpha^{n}} .
$$

Thus, we get

$$
\left|\ell \log \delta+\log (\sqrt{5} / 2)-n \log \alpha-\log \left(1+\alpha^{m-n}\right)\right|<\frac{23}{\alpha^{n}} .
$$

In order to find upper bounds for $n$ and $\ell$, we use a result of E. M. Matveev on lower bounds for nonzero linear forms in logarithms of algebraic numbers.

Let $\eta$ be an algebraic number of degree $d$ over $\mathbb{Q}$ with minimal primitive polynomial over the integers

$$
f(X):=a_{0} \prod_{i=1}^{d}\left(X-\eta^{(i)}\right) \in \mathbb{Z}[X],
$$

where the leading coefficient $a_{0}$ is positive. The logarithmic height of $\eta$ is given by

$$
h(\eta):=\frac{1}{d}\left(\log a_{0}+\sum_{i=1}^{d} \log \max \left\{\left|\eta^{(i)}\right|, 1\right\}\right) .
$$


In this work we will use the following properties. If $\eta=p / q$ is a rational number with $\operatorname{gcd}(p, q)=1$ and $q>0$, then $h(\eta)=\log \max \{|p|, q\}$. Are also known: $h\left(\eta^{s}\right)=|s| h(\eta)$ for all $s \in \mathbb{Z}$ and

$$
h(\eta \pm \gamma) \leq h(\eta)+h(\gamma)+\log 2, \quad h\left(\eta \gamma^{ \pm 1}\right) \leq h(\eta)+h(\gamma) .
$$

Our main tool is a lower bound for a linear form in logarithms of algebraic numbers given by the following result of Matveev [10]:

Theorem 1 (Matveev's theorem). Let $\mathbb{L} \subseteq \mathbb{R}$ be a real algebraic number field of degree $d_{\mathbb{L}}$ over $\mathbb{Q}, \eta_{1}, \ldots, \eta_{l}$ non-zero elements of $\mathbb{L}$, and $d_{1}, \ldots, d_{l}$ rational integers. Put

$$
\Lambda:=\eta_{1}^{d_{1}} \cdots \eta_{l}^{d_{l}}-1 \quad \text { and } \quad D \geq \max \left\{\left|d_{1}\right|, \ldots,\left|d_{l}\right|, 3\right\} .
$$

Let $A_{i} \geq \max \left\{d_{\mathbb{L}} h\left(\eta_{i}\right),\left|\log \eta_{i}\right|, 0.16\right\}$ be real numbers, for $i=1, \ldots, l$. Then, assuming that $\Lambda \neq 0$, we have

$$
|\Lambda|>\exp \left(-3 \times 30^{l+3} \times l^{4.5} \times d_{\mathbb{L}}^{2}\left(1+\log d_{\mathbb{L}}\right)(1+\log D) A_{1} \cdots A_{l}\right) .
$$

We apply Matveev's theorem on the left-hand side of (12). We take $l:=4$,

$$
\begin{array}{rll}
\eta_{1}:=\delta, & \eta_{2}:=\sqrt{5} / 2, \quad \eta_{3}:=\alpha, \quad \eta_{4}:=1+\alpha^{m-n}, \\
d_{1}:=\ell, & d_{2}:=1, \quad d_{3}:=-n, \quad d_{4}:=-1 .
\end{array}
$$

Furthermore, $\mathbb{L}=\mathbb{Q}(\sqrt{d}, \sqrt{5})$ which has degree $d_{\mathbb{L}}=4$. Since $\ell<n$, we take $D:=n$. We have $h\left(\eta_{1}\right)=(1 / 2) \log \delta, h\left(\eta_{2}\right)=(1 / 2) \log 5, h\left(\eta_{3}\right)=(1 / 2) \log \alpha$ and

$$
\begin{aligned}
h\left(\eta_{4}\right) & \leq h(1)+h\left(\alpha^{m-n}\right)+\log 2 \\
& =(n-m) h(\alpha)+\log 2 \\
& =(n-m)\left(\frac{1}{2} \log \alpha\right)+\log 2 .
\end{aligned}
$$

Thus, we can take

$$
A_{1}=2 \log \delta, \quad A_{2}=2 \log 5, \quad A_{3}=2 \log \alpha, \quad A_{4}=(2 \log \alpha)(n-m)+4 \log 2 .
$$

Note that $\Gamma_{1} \neq 0$, since otherwise

$$
\delta^{\ell}=(2 / \sqrt{5})\left(\alpha^{n}+\alpha^{m}\right) \in \mathbb{Q}(\sqrt{5}) .
$$

But given that $d \neq 5$ is squarefree, it follows that $\mathbb{Q}(\sqrt{d}) \cap \mathbb{Q}(\sqrt{5})=\mathbb{Q}$. Hence, $\ell=0$ and $\alpha^{n}+\alpha^{m}=(\sqrt{5} / 2)<2$, which is not possible for any $n>m \geq 0$.

Now Matveev's Theorem 1 tells us that

$$
\begin{aligned}
\log \left|\Gamma_{1}\right| & >-1.4 \cdot 30^{7} 4^{4.5} 4^{2}(1+\log 4)(1+\log n)(2 \log \delta)(2 \log 5)(2 \log \alpha) \\
& \cdot((2 \log \alpha)(n-m)+4 \log 2) \\
> & -7.2 \times 10^{15}(n-m)(\log n)(\log \delta) .
\end{aligned}
$$

Comparing the above inequality with (11), we get

$$
n \log \alpha-\log 23<7.2 \times 10^{15}(n-m)(\log n)(\log \delta) .
$$


Thus,

$$
n<1.5 \times 10^{16}(n-m)(\log n)(\log \delta) .
$$

This inequality was under the assumption that $n \geq 3$, but if $n=2$, then the above inequality obviously holds as well

Returning to equation $F_{m}+F_{n}=X_{\ell}$, and rewriting it as

$$
\frac{\alpha^{n}}{\sqrt{5}}-\frac{1}{2} \delta^{\ell}=\frac{1}{2} \eta^{\ell}+\frac{\beta^{n}}{\sqrt{5}}-F_{m},
$$

we obtain

$$
\left|\delta^{\ell}(\sqrt{5} / 2) \alpha^{-n}-1\right|<\frac{1.1}{\alpha^{n-m}} .
$$

Put

$$
\Lambda_{2}:=\delta^{\ell}(\sqrt{5} / 2) \alpha^{-n}-1, \quad \Gamma_{2}:=\ell \log \delta+\log (\sqrt{5} / 2)-n \log \alpha .
$$

Given that $n-m \geq 2$, we have that $\left|\Lambda_{2}\right|=\left|e^{\Gamma_{2}}-1\right|<0.421$. It follows that

$$
|\ell \log \delta+\log (\sqrt{5} / 2)-n \log \alpha|=\left|\Lambda_{2}\right|<e^{\left|\Gamma_{2}\right|}\left|e^{\Gamma_{2}}-1\right|<\frac{2}{\alpha^{n-m}} .
$$

Furthermore, $\Gamma_{2} \neq 0$, since $\delta^{\ell} \notin \mathbb{Q}(\sqrt{5})$ by a previous argument and $\alpha^{n}>2$ for all $n \geq 2$.

Applying Matveev's Theorem 1 with the parameters $l:=3$,

$$
\eta_{1}:=\delta, \quad \eta_{2}:=\sqrt{5} / 2, \quad \eta_{3}:=\alpha, \quad d_{1}:=\ell, \quad d_{2}:=1, \quad d_{3}:=-n,
$$

we can conclude that

$$
\log \left|\Gamma_{2}\right|>-1.5 \cdot 10^{14}(\log \delta)(\log n)(\log \alpha),
$$

and comparing with (15), we get

$$
n-m<3.2 \cdot 10^{14}(\log \delta)(\log n) .
$$

We replace the previous bound (16) on $n-m$ in (13) and use the fact that $\delta^{\ell} \leq \alpha^{n+1}\left(1+\alpha^{-2}\right)$, to obtain bounds on $n$ and $\ell$ in terms of $\log n$ and $\log \delta$.

Let us record what we have proved so far.

Lemma 1. Let $(m, n, \ell)$ be a solution of $F_{m}+F_{n}=X_{\ell}$ with $n-m \geq 2, m \geq 0$ and $d \neq 5$, then

$$
\ell<1.6 \times 10^{30}(\log n)^{2}(\log \delta) \quad \text { and } \quad n<4.8 \times 10^{30}(\log n)^{2}(\log \delta)^{2} .
$$

\section{Absolute bounds}

In this section we will find absolute bounds for $m, n$ and $\ell$, which determine that (8) only has a finite number of solutions.

We recall that $(m, n, \ell)=\left(m_{i}, n_{i}, \ell_{i}\right)$, where $n_{i}-m_{i} \geq 2, m_{i} \geq 0$, so $n_{i} \geq 2$, for $i=1,2$. Moreover, $1 \leq \ell_{1}<\ell_{2}$. We return to inequality (15) and write:

$$
\left|\Gamma_{2}^{(i)}\right|:=\left|\ell_{i} \log \delta+\log (\sqrt{5} / 2)-n_{i} \log \alpha\right|<\frac{2}{\alpha^{n_{i}-m_{i}}}, \quad \text { for } \quad i=1,2
$$


We make a suitable cross product between $\Gamma_{2}^{(1)}, \Gamma_{2}^{(2)}$ and $\ell_{1}, \ell_{2}$ to eliminate the term involving $\log \delta$ in the above linear forms in logarithms:

$$
\begin{aligned}
\left|\Gamma_{3}\right|:=\left|\left(\ell_{2}-\ell_{1}\right) \log (\sqrt{5} / 2)+\left(\ell_{1} n_{2}-\ell_{2} n_{1}\right) \log \alpha\right| & =\left|\ell_{2} \Gamma_{2}^{(1)}-\ell_{1} \Gamma_{2}^{(2)}\right| \\
& \leq \ell_{2}\left|\Gamma_{2}^{(2)}\right|+\ell_{1}\left|\Gamma_{2}^{(2)}\right| \\
& \leq \frac{2 \ell_{2}}{\alpha^{n_{1}-m_{1}}}+\frac{2 \ell_{1}}{\alpha^{n_{2}-m_{2}}} \\
& \leq \frac{4 n_{2}}{\alpha^{\lambda}}
\end{aligned}
$$

with $\lambda:=\min _{i=1,2}\left\{n_{i}-m_{i}\right\}$.

Next, we apply Matveev's theorem with $l=2$,

$$
\eta_{1}:=2 a, \quad \eta_{2}:=\alpha, \quad d_{1}:=\ell_{1}-\ell_{2}, \quad d_{2}:=\ell_{1} n_{2}-\ell_{2} n_{1} .
$$

We take $\mathbb{L}:=\mathbb{Q}(\sqrt{5})$ and $d_{\mathbb{L}}:=2$. We continue by remarking that $\Gamma_{3} \neq 0$, because $\alpha$ is a unit in the ring of algebraic integers of $\mathbb{Q}(\sqrt{5})$ while the norm of $\sqrt{5} / 2$ is $5 / 4$.

Note that $\left|\ell_{2}-\ell_{1}\right|<\ell_{2}<n_{2}$. Further, from inequality (18), we have

$$
\left|\ell_{1} n_{2}-\ell_{2} n_{1}\right|<\left(\ell_{2}-\ell_{1}\right) \frac{\log (\sqrt{5} / 2)}{\log \alpha}+\frac{4 \ell_{2}}{\alpha^{\lambda} \log \alpha}<3.4 \ell_{2}<3.4 n_{2}
$$

given that $\lambda \geq 2$. So, we can take $D:=3.4 n_{2}$.

From Matveev's theorem

$$
\log \left|\Gamma_{3}\right|>-2.6 \cdot 10^{10}\left(\log n_{2}\right)(\log \alpha)
$$

Combining this with (18), we get

$$
\lambda<2.7 \cdot 10^{10} \log n_{2} .
$$

Without loss generality, we can assume that $\lambda=n_{i}-m_{i}$, for $i \in\{1,2\}$ fixed.

We set $\{i, j\}=\{1,2\}$ and return to (12) to replace $(m, n, \ell)=\left(m_{i}, n_{i}, \ell_{i}\right)$ :

$$
\left|\Gamma_{1}^{(i)}\right|=\left|\ell_{i} \log \delta+\log (\sqrt{5} / 2)-n_{i} \log \alpha-\log \left(1+\alpha^{-\left(n_{i}-m_{i}\right)}\right)\right|<\frac{23}{\alpha^{n_{i}}}
$$

then to (15), with $(m, n, \ell)=\left(m_{j}, n_{j}, \ell_{j}\right)$ :

$$
\left|\Gamma_{2}^{(j)}\right|=\left|\ell_{j} \log \delta+\log (\sqrt{5} / 2)-n_{j} \log \alpha\right|<\frac{2}{\alpha^{n_{j}-m_{j}}} .
$$

We perform a cross product in inequalities (20) and (21) in order to eliminate the term $\log \delta$ :

$$
\begin{aligned}
\left|\Gamma_{4}\right| & :=\left|\left(\ell_{i}-\ell_{j}\right) \log (\sqrt{5} / 2)+\left(n_{i} \ell_{j}-n_{j} \ell_{i}\right) \log \alpha+\ell_{j} \log \left(1+\alpha^{-\left(n_{i}-m_{i}\right)}\right)\right| \\
& =\left|\ell_{i} \Gamma_{2}^{(j)}-\ell_{j} \Gamma_{1}^{(i)}\right| \leq \ell_{i}\left|\Gamma_{2}^{(j)}\right|+\ell_{j}\left|\Gamma_{1}^{(i)}\right| \leq \frac{25 n_{2}}{\alpha^{\rho}}
\end{aligned}
$$

with $\rho:=\min \left\{n_{i}, n_{j}-m_{j}\right\}$.

If $\Gamma_{4}=0$, we then obtain

$$
(\sqrt{5} / 2)^{\ell_{i}-\ell_{j}}=\alpha^{n_{i} \ell_{j}-n_{j} \ell_{i}}\left(1+\alpha^{-\lambda}\right)^{\ell_{j}} .
$$


Since $\alpha$ is a unit, the right-hand side above is an algebraic integer. This is impossible because $\ell_{1}<\ell_{2}$ so $\ell_{i}-\ell_{j} \neq 0$, and neither $\sqrt{5} / 2$ nor $(\sqrt{5} / 2)^{-1}$ are algebraic integers. Hence, $\Gamma_{4} \neq 0$.

By using Matveev's theorem, with the parameters $l:=3$ and

$$
\begin{aligned}
\eta_{1}:=\sqrt{5} / 2, \quad \eta_{2}:=\alpha, \quad \eta_{3}:=1+\alpha^{-\lambda}, \\
d_{1}:=\ell_{i}-\ell_{j}, \quad d_{2}:=n_{i} \ell_{j}-n_{j} \ell_{i}, \quad d_{3}:=\ell_{j},
\end{aligned}
$$

and inequalities (19) and (22), we get

$$
\rho=\min \left\{n_{i}, n_{j}-m_{j}\right\}<6.8 \cdot 10^{12} \lambda \log n_{2}<2 \cdot 10^{22}\left(\log n_{2}\right)^{2} .
$$

Note that the instance $(i, j)=(2,1)$ leads to $n_{1}-m_{1} \leq n_{1} \leq n_{2}+2$ while $(i, j)=(1,2)$ lead to $\rho=\min \left\{n_{1}, n_{2}-m_{2}\right\}$. Hence, either the minimum is $n_{1}$, so

$$
n_{1}<2 \cdot 10^{22}\left(\log n_{2}\right)^{2},
$$

or the minimum is $n_{j}-m_{j}$ and from inequality (19) we get

$$
\max _{i=1,2}\left\{n_{i}-m_{i}\right\}<2 \cdot 10^{22}\left(\log n_{2}\right)^{2} .
$$

Next, assume that we are in case (24). We evaluate (20) in $i=1,2$ and make a new cross product in order to eliminate the term involving $\log \delta$ :

$$
\begin{aligned}
\left|\Gamma_{5}\right| & :=\mid\left(\ell_{1}-\ell_{2}\right) \log (\sqrt{5} / 2)+\left(n_{1} \ell_{2}-n_{2} \ell_{1}\right) \log \alpha \\
& +\ell_{2} \log \left(1+\alpha^{m_{1}-n_{1}}\right)-\ell_{1} \log \left(1+\alpha^{m_{2}-n_{2}}\right) \mid \\
& =\left|\ell_{1} \Gamma_{1}^{(2)}-\ell_{2} \Gamma_{1}^{(1)}\right| \leq \ell_{1}\left|\Gamma_{1}^{(2)}\right|+\ell_{2}\left|\Gamma_{1}^{(1)}\right| \\
& <\frac{46 n_{2}}{\alpha^{n_{1}-2}} .
\end{aligned}
$$

In the above inequality we used inequality (10) to conclude that $\min \left\{n_{1}, n_{2}\right\} \geq n_{1}-2$. In order to apply Matveev's theorem we will prove that $\Gamma_{5} \neq 0$.

Lemma 2. The equation

$$
(\sqrt{5} / 2)^{\ell_{2}-\ell_{1}}=\alpha^{m_{1} \ell_{2}-m_{2} \ell_{1}}\left(1+\alpha^{n_{1}-m_{1}}\right)^{\ell_{2}}\left(1+\alpha^{n_{2}-m_{2}}\right)^{-\ell_{1}}
$$

has no solution in integers $1 \leq \ell_{1}<\ell_{2}$ and $n_{i}-m_{i} \geq 2, m_{i} \geq 0$ for $i=1,2$.

Proof. We let $\mathbb{K}=\mathbb{Q}(\sqrt{5})$. For any positive integer $k$

$$
N_{\mathbb{K} / \mathbb{Q}}\left(1+\alpha^{k}\right)=\left(1+\alpha^{k}\right)\left(1+\beta^{k}\right)=1+(-1)^{k}+\alpha^{k}+\beta^{k}
$$

so

$$
N_{\mathbb{K} / \mathbb{Q}}\left(1+\alpha^{k}\right)=\left\{\begin{array}{cccc}
L_{k}, & \text { if } & k \equiv 1 \quad(\bmod 2), \\
5 F_{k / 2}^{2}, & \text { if } & k \equiv 2 \quad(\bmod 4), \\
L_{k / 2}^{2}, & \text { if } & k \equiv 0 & (\bmod 4) .
\end{array}\right.
$$

Hence, assuming (26) and taking norms we get

$$
\begin{aligned}
\left(\frac{-5}{4}\right)^{\ell_{2}-\ell_{1}} & =N_{\mathbb{K} / \mathbb{Q}}(\sqrt{5} / 2)^{\ell_{2}-\ell_{1}} \\
& =N_{\mathbb{K} / \mathbb{Q}}(\alpha)^{m_{1} \ell_{2}-m_{2} \ell_{1}} \frac{N_{\mathbb{K} / \mathbb{Q}}\left(1+\alpha^{n_{1}-m_{1}}\right)^{\ell_{2}}}{N_{\mathbb{K} / \mathbb{Q}}\left(1+\alpha^{n_{2}-m_{2}}\right)^{\ell_{1}}} \\
& =(-1)^{m_{1} \ell_{2}-m_{2} \ell_{1}} \frac{E_{n_{1}-m_{1}}^{\ell_{2}}}{E_{n_{2}-m_{2}}^{\ell_{1}}}
\end{aligned}
$$


where $E_{k} \in\left\{L_{k}, L_{k / 2}^{2}, 5 F_{k / 2}^{2}\right\}$ according to the residue class of $k$ modulo 4 . If $n_{1}-m_{1}=n_{2}-m_{2}$, then the right-hand side is $E_{n_{1}-m_{1}}^{\ell_{2}-\ell_{1}}$, which is an integer. This is impossible since the left-hand side is not an integer. So, $n_{1}-m_{1} \neq n_{2}-m_{2}$. In the left, we have 5 in the numerator. Thus, we must have 5 in the numerator in the right as well. Since $5 \nmid L_{k}$ for any $k$, it follows that $n_{1}-m_{1} \equiv 2(\bmod 4)$ and $E_{n_{1}-m_{1}}=5 F_{\left(n_{1}-m_{1}\right) / 2}^{2}$. Thus, the exponent of 5 in $E_{n_{1}-m_{1}}^{\ell_{2}}$ is at least $\ell_{2}$. Since it is $\ell_{2}-\ell_{1}<\ell_{2}$ in the left it follows that $5 \mid E_{n_{2}-m_{2}}$. By the previous argument, $n_{2}-m_{2} \equiv 2(\bmod 4)$ and $E_{n_{2}-m_{2}}=5 F_{\left(n_{2}-m_{2}\right) / 2}^{2}$. By the Carmichael primitive divisor theorem, if $\ell \geq 7$ is odd, $F_{\ell}$ has a primitive prime factor $p$ which exceeds 5 and does not divide $F_{m}$ for any $m<\ell$. Using this theorem, we conclude easily that $\left(n_{1}-m_{1}\right) / 2 \leq 5$ and $\left(n_{2}-m_{2}\right) / 2 \leq 5$ (otherwise, since $\left(n_{1}-m_{1}\right) / 2 \neq\left(n_{2}-m_{2}\right) / 2$ are odd, the fraction $\left(5 F_{\left(n_{1}-m_{1}\right) / 2}\right)^{\ell_{2}} /\left(5 F_{\left(n_{2}-m_{2}\right) / 2}^{2}\right)^{\ell_{1}}$ in reduced form will contain with positive or negative exponent a primitive prime $p>5$ of $F_{k}$, where $k=\max \left\{\left(n_{1}-m_{1}\right) / 2,\left(n_{2}-m_{2}\right) / 2\right\}$, which does not appear in the left).

Assume that one of $\left(n_{1}-m_{1}\right) / 2$ or $\left(n_{1}-m_{2}\right) / 2$ is 5 . Then the exponent of 5 is one of $3 \ell_{2}$ (if $\left(n_{1}-m_{1}\right) / 2=5$ ), or $\ell_{2}-3 \ell_{1}$ (if $\left.\left(n_{2}-m_{2}\right) / 2=5\right)$ and none of these equals $\ell_{2}-\ell_{1}$ which is the exponent of 5 on the left. Hence, $\left\{\left(n_{1}-m_{1}\right) / 2,\left(n_{2}-m_{2}\right) / 2\right\}=\{1,3\}$. Since the exponent of 2 appears with negative sign in the left, we conclude that the only possibility is

$$
\left(n_{1}-m_{1}\right) / 2=1, \quad\left(n_{2}-m_{2}\right) / 2=3 .
$$

In this case, we get

$$
\left(\frac{-5}{4}\right)^{\ell_{2}-\ell_{1}}=(-1)^{m_{1} \ell_{2}-m_{2} \ell_{1}}\left(\frac{5^{\ell_{2}}}{(5 \cdot 4)^{\ell_{1}}}\right)= \pm \frac{5^{\ell_{2}-\ell_{1}}}{4^{\ell_{1}}}
$$

and comparing the exponents of 2 in both sides we get $\ell_{2}-\ell_{1}=\ell_{1}$, so $\ell_{2}=2 \ell_{1}$. We now return to equation (26) and use

$$
1+\alpha^{2}=\sqrt{5} \alpha \quad \text { and } \quad 1+\alpha^{6}=2 \sqrt{5} \alpha^{3},
$$

to get

$$
\begin{aligned}
\left(\frac{\sqrt{5}}{2}\right)^{\ell_{1}} & =\left(\frac{\sqrt{5}}{2}\right)^{\ell_{2}-\ell_{1}}=\alpha^{m_{1} \ell_{2}-m_{2} \ell_{1}}\left(\frac{(\sqrt{5} \alpha)^{\ell_{2}}}{\left(2 \sqrt{5} \alpha^{3}\right)^{\ell_{1}}}\right) \\
& =\alpha^{\ell_{1}\left(2 m_{1}-m_{2}\right)} \sqrt{5}^{\ell_{2}-\ell_{1}} \alpha^{\ell_{2}-3 \ell_{1}} 2^{-\ell_{1}}=\left(\alpha^{2 m_{1}-m_{2}-1}\left(\frac{\sqrt{5}}{2}\right)\right)^{\ell_{1}} .
\end{aligned}
$$

Extracting $\ell_{1}$ powers, we get that

$$
\frac{\sqrt{5}}{2}=\zeta \alpha^{2 m_{1}-m_{2}-1} \frac{\sqrt{5}}{2},
$$

where $\zeta$ is some root of unity of order $2 \ell_{1}$. Hence, $\alpha^{2 m_{1}-m_{2}-1}=\zeta^{-1}$. Since the left-hand side is real and positive, we have $\zeta^{-1}=1$ so $2 m_{1}-m_{2}-1=0$. Since $n_{1}=m_{1}+2, n_{2}=m_{2}+6$, we get that

$$
n_{2}=m_{2}+6=\left(2 m_{1}-1\right)+6=2\left(n_{1}-2\right)+5=2 n_{1}+1 .
$$

Hence, putting $k=n_{1}$, we have gotten to the situation where

$$
\begin{aligned}
& X_{\ell_{1}}=F_{n_{1}}+F_{m_{1}}=F_{n_{1}}+F_{n_{1}-2}, \\
& X_{\ell_{2}}=X_{2 \ell_{1}}=F_{n_{2}}+F_{m_{2}}=F_{2 n_{1}+1}+F_{2 n_{1}-5} .
\end{aligned}
$$


Since

$$
X_{2 \ell_{1}}=2 X_{\ell_{1}}^{2} \pm 1
$$

we get

$$
F_{2 n_{1}+1}+F_{2 n_{1}-5}=X_{2 \ell_{1}}=2 X_{\ell_{1}}^{2} \pm 1=2\left(F_{n_{1}}+F_{n_{1}-2}\right)^{2} \pm 1 .
$$

Of course this is absurd because the left-hand side is always even and the right-hand side is always odd. Thus, it is not possible that

$$
F_{2 n_{1}+1}+F_{2 n_{1}-5}-2\left(F_{n_{1}}+F_{n_{1}-2}\right)^{2}= \pm 1 .
$$

It is an easy exercise though to show that the left-hand side above is \pm 4 (namely, $(-1)^{n_{1}} 4$ for all $\left.n_{1} \geq 3\right)$.

We apply a linear form in four logarithms to obtain an upper bound to $n_{1}$. We take

$$
\begin{aligned}
& \eta_{1}:=\sqrt{5} / 2, \quad \eta_{2}:=\alpha, \quad \eta_{3}:=1+\alpha^{m_{1}-n_{1}}, \quad \eta_{4}:=1+\alpha^{m_{2}-n_{2}}, \\
& d_{1}:=\ell_{1}-\ell_{2}, \quad d_{2}:=n_{1} \ell_{2}-n_{2} \ell_{1}, \quad d_{3}:=\ell_{2}, \quad d_{4}:=-\ell_{1},
\end{aligned}
$$

and apply Matveev's theorem on the left-hand side of inequalities (25). Combining the resulting inequality with the right-hand side in (25) and inequalities (19) and (24) leads us to

$$
\begin{aligned}
n_{1}< & 2.12 \cdot 10^{15} h\left(1+\alpha^{m_{1}-n_{1}}\right) h\left(1+\alpha^{m_{2}-n_{2}}\right)\left(\log n_{2}\right) \\
& <5 \cdot 10^{14}\left(n_{1}-m_{1}\right)\left(n_{2}-m_{2}\right)\left(\log n_{2}\right) \\
& <2.7 \cdot 10^{47}\left(\log n_{2}\right)^{4}
\end{aligned}
$$

Thus, we have that inequality (27) holds provided that (24) holds. Otherwise, (23) holds which is even better than (27). Hence, we conclude that $n_{1}<2.7 \cdot 10^{47}\left(\log n_{2}\right)^{4}$ holds in all cases.

By inequality (9),

$$
\log \delta \leq \ell_{1} \log \delta \leq\left(n_{1}+1\right) \log \alpha+\log \left(1+\alpha^{-2}\right)<1.3 \cdot 10^{47}\left(\log n_{2}\right)^{4} .
$$

Putting this into (17) we get $n_{2}<8.2 \cdot 10^{124}\left(\log n_{2}\right)^{10}$, and then $n_{2}<2.1 \cdot 10^{150}$.

In summary, we have proved the following result.

Lemma 3. Let $\left(m_{i}, n_{i}, \ell_{i}\right)$ be two solutions of $F_{m_{i}}+F_{n_{i}}=X_{\ell_{i}}$ for $i=1,2$, with $n_{i}-m_{i} \geq 2$, $m_{i} \geq 0, d \neq 5,1 \leq \ell_{1}<\ell_{2}$, then

$$
\max \left\{m_{1}, \ell_{1}\right\}<n_{1}<4 \cdot 10^{57} \quad \text { and } \quad \max \left\{m_{2}, \ell_{2}\right\}<n_{2}<2.1 \cdot 10^{150} .
$$

\section{$5 \quad$ Reducing $n_{1}$ and $n_{2}$}

In the above Lemma 3, we obtained upper bounds on our variables which are very large, so we need to reduce them. With this aim, we use some results from the theory of continued fractions and the geometry of numbers.

The following results, well-known in the theory of Diophantine approximation, will be used for the treatment of linear forms homogeneous in two integer variables. 
Lemma 4. Let $\tau$ be an irrational number, $M$ be a positive integer and $p_{0} / q_{0}, p_{1} / q_{1}, \ldots$ be all the convergents of the continued fraction of $\tau$. Let $N$ be such that $q_{N}>M$. Then putting

$$
a(M):=\max \left\{a_{t}: t=0,1, \ldots, N\right\}, \quad \text { the inequality } \quad|m \tau-n|>\frac{1}{(a(M)+2) m},
$$

holds for all pairs $(n, m)$ of integers with $0<m<M$.

For the treatment of nonhomogeneous linear forms in two integer variables, we will use a slight variation of a result due to Dujella and Pethő, which itself is a generalization of a result of Baker and Davenport (see [4]). For a real number $X$, we put

$$
\|X\|:=\min \{|X-n|: n \in \mathbb{Z}\}
$$

for the distance from $X$ to the nearest integer.

Lemma 5. Let $\tau$ be an irrational number, $M$ be a positive integer, and $p / q$ be a convergent of the continued fraction of the irrational $\tau$ such that $q>6 M$. Let $A, B, \mu$ be some real numbers with $A>0$ and $B>1$. Put $\epsilon:=\|\mu q\|-M\|\tau q\|$. If $\epsilon>0$, then there is no solution to the inequality

$$
0<|m \tau-n+\mu|<A B^{-k}
$$

in positive integers $m, n$ and $k$ with

$$
m \leq M \quad \text { and } \quad k \geq \frac{\log (A q / \epsilon)}{\log B} .
$$

At various occasions, we need to find a lower bound for linear forms with bounded integer coefficients (in three and four integer variables). Let $\tau_{1}, \ldots, \tau_{t} \in \mathbb{R}$ and the linear form

$$
x_{1} \tau_{1}+x_{2} \tau_{2}+\cdots+x_{t} \tau_{t} \quad \text { with } \quad\left|x_{i}\right| \leq X_{i} .
$$

We set $X:=\max \left\{X_{i}\right\}, C>(t X)^{t}$ and consider the integer lattice $\Omega$ generated by

$$
\mathbf{b}_{j}:=\mathbf{e}_{j}+\left\lfloor C \tau_{j}\right\rceil \mathbf{e}_{t} \quad \text { for } \quad 1 \leq j \leq t-1 \quad \text { and } \quad \mathbf{b}_{t}:=\left\lfloor C \tau_{t}\right\rceil \mathbf{e}_{t},
$$

where $C$ is a sufficiently large positive constant.

Lemma 6. Let $X_{1}, \ldots, X_{t}$ be positive integers such that $X:=\max \left\{X_{i}\right\}$ and $C>(t X)^{t}$ is a fixed constant. With the above notation on $\Omega$, we consider a reduced base $\left\{\mathbf{b}_{i}\right\}$ to $\Omega$ and its base of Gram-Schmidt $\left\{\mathbf{b}_{i}^{*}\right\}$ associated. We set

$$
c_{1}:=\max _{1 \leq i \leq t} \frac{\left\|\mathbf{b}_{1}\right\|}{\left\|\mathbf{b}_{i}^{*}\right\|}, \quad \delta:=\frac{\left\|\mathbf{b}_{1}\right\|}{c_{1}}, \quad Q:=\sum_{i=1}^{t-1} X_{i}^{2} \quad \text { and } \quad T:=\left(1+\sum_{i=1}^{t} X_{i}\right) / 2 .
$$

If the integers $x_{i}$ satisfy that $\left|x_{i}\right| \leq X_{i}$, for $i=1, \ldots, t$ and $\delta^{2} \geq T^{2}+Q$, then we have

$$
\left|\sum_{i=1}^{t} x_{i} \tau_{i}\right| \geq \frac{\sqrt{\delta^{2}-Q}-T}{C}
$$

For more details, see Proposition 2.3.20 in [1, Section 2.3.5]. 


\subsection{First reduction}

With this purpose of reducing the upper bound to $n_{1}$ and $n_{2}$ given in Lemma 3 to cases that can be treated computationally, we return to $\Gamma_{3}, \Gamma_{4}$ and $\Gamma_{5}$.

Dividing both sides of inequality (18) by $\left(\ell_{2}-\ell_{1}\right) \log \alpha$, we obtain

$$
\left|\frac{\log (\sqrt{5} / 2)}{\log \alpha}-\frac{\ell_{2} n_{1}-\ell_{1} n_{2}}{\ell_{2}-\ell_{1}}\right|<\frac{8.4 n_{2}}{\alpha^{\lambda}\left(\ell_{2}-\ell_{1}\right)} \quad \text { with } \quad \lambda:=\min _{i=1,2}\left\{n_{i}-m_{i}\right\} .
$$

Bellow we apply Lemma 4. We put $\tau:=\log (\sqrt{5} / 2) / \log \alpha$ (which is an irrational) and compute its continued fraction $\left[a_{0}, a_{1}, a_{2}, \ldots\right]$ and its convergents $p_{1} / q_{1}, p_{2} / q_{2}, \ldots$

$$
[0,4,3,5,7,3,1,8,45,1,3,1, \ldots] \quad \text { and } \quad 0, \frac{1}{4}, \frac{3}{13}, \frac{16}{69}, \frac{115}{496}, \frac{361}{1557}, \frac{476}{2053}, \ldots
$$

Furthermore, we note that taking $M:=2.1 \cdot 10^{150}$ (according to Lemma 3), it follows that

$$
q_{282}>M>n_{2}>\ell_{2}-\ell_{1} \quad \text { and } \quad a(M):=\max \left\{a_{i}: 0 \leq i \leq 282\right\}=258 .
$$

Then, by Lemma 4, we have that

$$
\left|\tau-\frac{\ell_{2} n_{1}-\ell_{1} n_{2}}{\ell_{2}-\ell_{1}}\right|>\frac{1}{260\left(\ell_{2}-\ell_{1}\right)^{2}} .
$$

Hence, combining the inequalities (29) and (30), we obtain

$$
\alpha^{\lambda}<2184 \cdot n_{2}\left(\ell_{2}-\ell_{1}\right)<9.7 \cdot 10^{303},
$$

so $\lambda \leq 1455$.

Now, for each $n_{i}-m_{i}=\lambda \in[2,1455]$ we estimate (via LLL-algorithm) a lower bound for $\left|\Gamma_{4}\right|$, with

$$
\Gamma_{4}=\left(\ell_{i}-\ell_{j}\right) \log (\sqrt{5} / 2)+\left(n_{i} \ell_{j}-n_{j} \ell_{i}\right) \log \alpha+\ell_{j} \log \left(1+\alpha^{m_{i}-n_{i}}\right)
$$

given in inequality (22). Recall that $\Gamma_{4} \neq 0$.

We put as in (28), $t:=3$,

$$
\tau_{1}:=\log (\sqrt{5} / 2), \quad \tau_{2}:=\log \alpha, \quad \tau_{3}:=\log \left(1+\alpha^{-\lambda}\right),
$$

and

$$
x_{1}:=\ell_{i}-\ell_{j}, \quad x_{2}:=n_{i} \ell_{j}-n_{j} \ell_{i}, \quad x_{3}:=\ell_{j} .
$$

Further, we set $X:=7.2 \cdot 10^{150}$ as an upper bound to $\left|x_{i}\right|<3.4 n_{2}$ for all $i=1,2,3$, and $C:=(20 X)^{5}$. A computer search allows us to conclude, together with inequality (22), that

$$
10^{-608}<\min _{\lambda \in[2,1455]}\left|\Gamma_{4}\right|<25 n_{2} \cdot \alpha^{-\rho}, \quad \text { with } \rho:=\min \left\{n_{i}, n_{j}-m_{j}\right\},
$$

which leads to $\rho \leq 3635$. As we noted before, $\rho=n_{1}$ (so $n_{1} \leq 3635$ ), or $\rho=n_{j}-m_{j}$.

Next we suppose that $n_{j}-m_{j}=\rho \leq 3635$. Since $\lambda \leq 1455$, we have

$$
\lambda=\min _{i=1,2}\left\{n_{i}-m_{i}\right\} \leq 1455 \quad \text { and } \quad \chi:=\max _{i=1,2}\left\{n_{i}-m_{i}\right\} \leq 3635 .
$$

Returning to inequality (25) which involves

$$
\begin{aligned}
\Gamma_{5} & :=\left(\ell_{1}-\ell_{2}\right) \log (\sqrt{5} / 2)+\left(n_{1} \ell_{2}-n_{2} \ell_{1}\right) \log \alpha \\
& +\ell_{2} \log \left(1+\alpha^{m_{1}-n_{1}}\right)-\ell_{1} \log \left(1+\alpha^{m_{2}-n_{2}}\right) \neq 0,
\end{aligned}
$$


we use again the LLL-algorithm to estimate a lower bound for $\left|\Gamma_{5}\right|$ and so to find a beter bound to $n_{1}$ than the one given in Lemma 3 .

We will distinguish the cases $\lambda<\chi$ or $\lambda=\chi$.

The case $\lambda<\chi$.

We take $\lambda \in[2,1455]$ and $\chi \in[\lambda+1,3635]$ and put for (28), $t:=4$,

$$
\tau_{1}:=\log (\sqrt{5} / 2), \quad \tau_{2}:=\log \alpha, \quad \tau_{3}:=\log \left(1+\alpha^{m_{1}-n_{1}}\right), \quad \tau_{4}:=\log \left(1+\alpha^{m_{2}-n_{2}}\right),
$$

and

$$
x_{1}:=\ell_{1}-\ell_{2}, \quad x_{2}:=n_{1} \ell_{2}-n_{2} \ell_{1}, \quad x_{3}:=\ell_{2}, \quad x_{4}:=-\ell_{1} .
$$

Also we put $X:=7.2 \cdot 10^{150}$ and $C:=(7 X)^{9}$. Computationally we confirm that,

$$
10^{-1215}<\min _{\substack{\lambda \in[2,1455] \\ \chi \in[\lambda+1,3635]}}\left|\Gamma_{5}\right|,
$$

which together with inequality (25) lead to inequality

$$
\alpha^{n_{1}-2}<46 \cdot 10^{1215} n_{2} .
$$

Hence, considering the upper bound on $n_{2}$ given in Lemma 3, we conclude that $n_{1} \leq 6545$.

The case $\lambda=\chi$.

In this case, we have

$$
\Gamma_{5}:=\left(\ell_{2}-\ell_{1}\right)\left(\log (2 / \sqrt{5})+\log \left(1+\alpha^{m_{1}-n_{1}}\right)\right)+\left(n_{1} \ell_{2}-n_{2} \ell_{1}\right) \log \alpha .
$$

We divide inequality (25) by $\left(\ell_{2}-\ell_{1}\right) \log \alpha$ to obtain

$$
\left|\frac{\left|\log (2 / \sqrt{5})+\log \left(1+\alpha^{m_{1}-n_{1}}\right)\right|}{\log \alpha}-\frac{\ell_{2} n_{1}-\ell_{1} n_{2}}{\ell_{2}-\ell_{1}}\right|<\frac{96 n_{2}}{\alpha^{n_{1}-2}\left(\ell_{2}-\ell_{1}\right)} .
$$

We now put $\tau_{\lambda}:=\left|\log (2 / \sqrt{5})+\log \left(1+\alpha^{\lambda}\right)\right| / \log \alpha$ and compute its continued fractions $\left[a_{0}^{(\lambda)}, a_{1}^{(\lambda)}, a_{2}^{(\lambda)}, \ldots\right]$ and its convergents $p_{1}^{(\lambda)} / q_{1}^{(\lambda)}, p_{2}^{(\lambda)} / q_{2}^{(\lambda)}, \ldots$ for each $\lambda \in[2,1455]$. Furthermore, for each case we find an integer $t_{\lambda}$ such that $q_{t_{\lambda}}^{(\lambda)}>2.1 \cdot 10^{150}>n_{2}>\ell_{2}-\ell_{1}$ and calculate

$$
a(M):=\max _{2 \leq \lambda \leq 1455}\left\{a_{i}^{(\lambda)}: 0 \leq i \leq t_{\lambda}\right\} .
$$

A simple computational routine in Mathematica reveals that for $\lambda=312, t_{\lambda}=270$ and $i=223$ we have $a(M)=a_{223}^{(312)}=1000002$. Hence, combining the concusion of Lemma 4 and inequality (33), we get $\alpha^{n_{1}-2}<96 \cdot 1000004 n_{2}\left(\ell_{2}-\ell_{1}\right)<4.3 \cdot 10^{308}$, so $n_{1} \leq 1170$.

Hence, we obtain that $n_{1} \leq 6545$ holds in all cases $\left(\rho=n_{1}, \lambda<\chi\right.$ or $\left.\lambda=\chi\right)$. By inequality (9),

$$
\log \delta \leq \ell_{1} \log \delta \leq n_{1} \log \alpha+\log \left(1+\alpha^{-2}\right)<3150 .
$$

Considering the above inequality in (17) we conclude that $n_{2}<3 \cdot 10^{37}\left(\log n_{2}\right)^{2}$ which yield $n_{2}<4.4 \cdot 10^{41}$. In summary, after this first cycle of reduction, we have

$$
n_{1} \leq 6545 \quad \text { and } \quad n_{2}<4.4 \cdot 10^{41} .
$$


We note that the above upper bound for $n_{2}$ represents a very good reduction of the bound given in Lemma 3. Hence, it is expected that if we restart our reduction cycle with our new bound on $n_{2}$, then we can get an even better bound on $n_{1}$. Indeed, returning to (29), we take $M:=4.4 \cdot 10^{41}$ and computationally we verify that $q_{89}>M>n_{2}>\ell_{2}-\ell_{1}$ and $a(M):=\max \left\{a_{i}: 0 \leq i \leq\right.$ $89\}=a_{73}=161$, from which it follows that $\lambda \leq 414$. We now return to (31), where putting $X:=1.5 \cdot 10^{42}$ and $C:=(7 X)^{5}$, we apply LLL-algorithm to $\lambda \in[2,414]$. This time we get $7.9 \cdot 10^{-174}<\min _{\lambda \in[2,414]}\left|\Gamma_{4}\right|$, then $\rho \leq 1035$. Continuing under the assumption $n_{j}-m_{j}=\rho \leq$ 1035 , we return to (32) and put $X:=1.5 \cdot 10^{42}, C:=(11 X)^{9}$ and $M:=4.4 \cdot 10^{41}$ for the cases $\lambda<\chi$ and $\lambda=\chi$. One can confirm computationally that

$$
2.7 \cdot 10^{-347}<\min _{\substack{\lambda \in[2,414] \\ \chi \in[\lambda+1,1035]}}\left|\Gamma_{5}\right| \quad \text { and } \quad a(M)=a_{45}^{(43)}=19362
$$

respectively and thus we obtain $n_{1} \leq 1870$. Running one more time the reduction cycle, we concluded that $n_{1} \leq 1811$.

In the next lemma we summarize the reductions achieved.

Lemma 7. Let $\left(m_{i}, n_{i}, \ell_{i}\right)$ be two solutions of $F_{m_{i}}+F_{n_{i}}=X_{\ell_{i}}$ for $i=1,2$, with $n_{i}-m_{i} \geq 2$, $m_{i} \geq 0, d \neq 5,1 \leq \ell_{1}<\ell_{2}$, then

$$
m_{1}<n_{1} \leq 1811, \quad \ell_{1} \leq 990 \text { and } n_{2}<3.3 \cdot 10^{40} .
$$

\subsection{Final reduction.}

From (5) and (6) and the fact that $\left(X_{1}, Y_{1}\right)$ is the smallest positive integer solution to the Pell equation $X^{2}-d Y^{2}= \pm 1$, we obtain

$$
\begin{aligned}
X_{\ell} & =\frac{1}{2}\left(\delta^{\ell}+\eta^{\ell}\right)=\frac{1}{2}\left(\left(X_{1}+\sqrt{d} Y_{1}\right)^{\ell}+\left(X_{1}-\sqrt{d} Y_{1}\right)^{\ell}\right) \\
& =\frac{1}{2}\left(\left(X_{1}+\sqrt{X_{1}^{2} \mp 1}\right)^{\ell}+\left(X_{1}-\sqrt{X_{1}^{2} \mp 1}\right)^{\ell}\right):=P_{\ell}^{ \pm}\left(X_{1}\right) .
\end{aligned}
$$

Thus, returning to the equation $F_{m_{1}}+F_{n_{1}}=X_{\ell_{1}}$, we consider the equations:

$$
P_{\ell_{1}}^{+}\left(X_{1}\right)=F_{m_{1}}+F_{n_{1}} \quad \text { and } \quad P_{\ell_{1}}^{-}\left(X_{1}\right)=F_{m_{1}}+F_{n_{1}},
$$

with $m_{1} \in[0,1811], n_{1} \in\left[m_{1}+2,1811\right]$ and $\ell_{1} \in[1,990]$.

A computer search on the above equations (35) shows that

\begin{tabular}{cccccc|ccccc} 
& $\left(n_{1}, m_{1}, \ell_{1}\right)$ & $X_{1}$ & $d$ & $Y_{1}$ & $\delta$ & $\left(n_{1}, m_{1}, \ell_{1}\right)$ & $X_{1}$ & $d$ & $Y_{1}$ & $\delta$ \\
\cline { 2 - 9 }$P_{\ell_{1}}^{+}:$ & $(5,3,2)$ & 2 & 3 & 1 & $2+\sqrt{3}$ & $(12,10,2)$ & 10 & 11 & 3 & $10+3 \sqrt{11}$ \\
& $(8,5,3)$ & 2 & 3 & 1 & $2+\sqrt{3}$ & $(13,6,2)$ & 11 & 30 & 2 & $11+2 \sqrt{30}$ \\
& $(11,6,2)$ & 7 & 12 & 2 & $7+4 \sqrt{3}$ & $(21,5,2)$ & 74 & 219 & 5 & $74+5 \sqrt{219}$ \\
& $(11,6,4)$ & 2 & 3 & 1 & $2+\sqrt{3}$ & & & & & \\
\hline
\end{tabular}

It easy to see that $\left(n_{1}, m_{1}, \ell_{1}, X_{1}\right)=\left(2,0, \ell_{1}, 1\right)$ too are solutions for all $\ell_{1} \in[1,990]$. However, these lead to $Y_{1}=0$, which is not of interest to us. On the other hand

\begin{tabular}{cccccc} 
& $\left(n_{1}, m_{1}, \ell_{1}\right)$ & $X_{1}$ & $d$ & $Y_{1}$ & $\delta$ \\
\cline { 2 - 6 }$P_{\ell_{1}}^{-}:$ & $(4,0,2)$ & 1 & 2 & 1 & $1+\sqrt{2}$ \\
& $(3,1,2)$ & 1 & 2 & 1 & $1+\sqrt{2}$ \\
& $(5,3,3)$ & 1 & 2 & 1 & $1+\sqrt{2}$ \\
\hline & $(23,12,2)$ & 120 & 14401 & 1 & $120+\sqrt{14401}$ \\
\hline
\end{tabular}


are the only solutions. We note that $7+4 \sqrt{3}=(1+\sqrt{3})^{2}$, so these come from the same Pell equation with $d=3$.

From the above tables, we are let to set

$$
\begin{array}{lll}
\delta_{1}:=2+\sqrt{3}, & \delta_{2}:=10+3 \sqrt{11}, & \delta_{3}:=11+2 \sqrt{30}, \\
\delta_{4}:=74+5 \sqrt{219}, & \delta_{5}:=1+\sqrt{2}, & \delta_{6}:=120+\sqrt{14401} .
\end{array}
$$

We work on the linear form in logarithms $\Gamma_{1}$ and $\Gamma_{2}$, in order to reduce the upper bound on $n_{2}$ given in Lemma 7 From inequality (15), for $(m, n, \ell)=\left(m_{2}, n_{2}, \ell_{2}\right)$, we write

$$
\left|\ell_{2} \frac{\log \delta_{s}}{\log \alpha}-n_{2}+\frac{\log (\sqrt{5} / 2)}{\log \alpha}\right|<4.2 \cdot \alpha^{-\left(n_{2}-m_{2}\right)}, \text { for } s=1,2, \ldots, 6 .
$$

We put

$$
\tau_{s}:=\frac{\log \delta_{s}}{\log \alpha}, \quad \mu_{s}:=\frac{\log (\sqrt{5} / 2)}{\log \alpha} \quad \text { and } \quad A_{s}:=4.2, \quad B_{s}:=\alpha .
$$

By the Gelfond-Schneider's theorem, we conclude that $\tau_{s}$ is transcendental (so irrational). Inequality (36) can be rewritten as

$$
0<\left|\ell_{2} \tau_{s}-n_{2}+\mu_{s}\right|<A_{s} B_{s}^{-\left(n_{2}-m_{2}\right)}, \text { for } s=1,2, \ldots, 6 \text {. }
$$

Now, we take $M:=3.3 \times 10^{40}$ which is an upper bound on $n_{2}$ (according to Lemma 7), and apply Lemma 5 to inequality (37). For each $\tau_{s}$ with $s=1, \ldots, 6$, we compute its continued fraction $\left[a_{0}^{(s)}, a_{1}^{(s)}, a_{2}^{(s)}, \ldots\right]$ and its convergents $p_{1}^{(s)} / q_{1}^{(s)}, p_{2}^{(s)} / q_{2}^{(s)}, \ldots$.

In each case, by means of computer search with Mathematica, we find an integer $t_{s}$ such that

$$
q_{t_{s}}^{(s)}>2 \times 10^{41}=6 M \quad \text { and } \quad \epsilon_{s}:=\left\|\mu_{s} q^{(s)}\right\|-M\left\|\tau_{s} q^{(s)}\right\|>0 .
$$

Finally we found the values of $h_{s}:=\left\lfloor\log \left(A_{s} q_{t_{2}}^{s} / \epsilon_{s}\right) / \log B_{s}\right\rfloor$ :

\begin{tabular}{c|cccccc}
$s$ & 1 & 2 & 3 & 4 & 5 & 6 \\
$t_{s}$ & 73 & 74 & 98 & 86 & 85 & 81 \\
$\epsilon_{s}$ & $>0.34$ & $>0.24$ & $>0.35$ & $>0.38$ & $>0.09$ & 0.37 \\
$h_{s}$ & 204 & 204 & 203 & 206 & 209 & 203
\end{tabular}.

Hence, the above $h_{s}$ correspond to upper bounds on $n_{2}-m_{2}$, for each $s=1, \ldots, 6$, according to Lemma 5 ,

Replacing $(m, n, \ell)=\left(m_{2}, n_{2}, \ell_{2}\right)$ in inequality (12), we can write

$$
\left|\ell_{2} \frac{\log \delta_{s}}{\log \alpha}-n_{2}+\frac{\log \left((\sqrt{5} / 2) /\left(1+\alpha^{-\left(n_{2}-m_{2}\right)}\right)\right)}{\log \alpha}\right|<47.8 \cdot \alpha^{-n_{2}}, \text { for } s=1,2, \ldots, 6
$$

We now put

$$
\tau_{s}:=\frac{\log \delta_{s}}{\log \alpha}, \quad A_{s}:=47.8, \quad B_{s}:=\alpha
$$

and

$$
\mu_{s, n_{2}-m_{2}}:=\frac{\log \left((\sqrt{5} / 2) /\left(1+\alpha^{-\left(n_{2}-m_{2}\right)}\right)\right)}{\log \alpha} .
$$


With the above parameters we rewrite (38) as

$$
0<\left|\ell_{2} \tau_{s}-n_{2}+\mu_{s, n_{2}-m_{2}}\right|<A_{s} B_{s}^{-n_{2}} \text {, for } s=1,2, \ldots, 6 .
$$

Bellow we apply again Lemma 5 to the above inequality (39), for

$$
s=1, \ldots, 6 \quad \text { and } \quad n_{2}-m_{2} \in\left[1, d_{s}\right], \quad \text { with } \quad M:=3.3 \times 10^{40} .
$$

Taking

$$
\epsilon_{s, n_{2}-m_{2}}:=\left\|\mu_{s} q^{\left(s, n_{2}-m_{2}\right)}\right\|-M\left\|\tau_{s} q^{\left(s, n_{2}-m_{2}\right)}\right\|
$$

and

$$
h_{s, n_{2}-m_{2}}:=\left\lfloor\log \left(A_{s} q^{\left(s, n_{2}-m_{2}\right)} / \epsilon_{s, n_{2}-m_{2}}\right) / \log B_{s}\right\rfloor,
$$

we obtain computationally that

$$
\max \left\{h_{s, n_{2}-m_{2}}: s=1, \ldots, 6 \text { and } n_{2}-m_{2}=1, \ldots, h_{s}\right\} \leq 227 .
$$

Thus, by Lemma 5. we have $n_{2} \leq 227$, for all $s=1, \ldots, 6$. Running a new reduction cycle from inequality (38), with $M:=227$ (new upper bound on $n_{2}$ ), we finally obtain $n_{2} \leq 42$ and by inequality (10) we have $n_{1} \leq n_{2}+2$. Given that $\delta^{\ell_{2}} \leq 2 \alpha^{n_{2}}$ we conclude that $\ell_{1}<\ell_{2} \leq 25$. Gathering all the information obtained, our problem is reduced to search solutions for (8) in the following range:

$$
1 \leq \ell_{1}<\ell_{2} \leq 25, \quad m_{2}+2 \leq n_{2} \in[2,42] \quad \text { and } \quad m_{1}+2 \leq n_{1} \in[2,44] .
$$

Checking equalities (8) in the above range, we obtain the following solutions.

For $\epsilon=+1$ :

$$
\begin{gathered}
F_{3}+F_{5}=7=X_{2}, \quad F_{5}+F_{8}=26=X_{3}, \quad F_{6}+F_{11}=97=X_{4} \quad(\delta=2+\sqrt{3}) \\
F_{3}+F_{6}=10=X_{1}, \quad F_{10}+F_{12}=199=X_{2}, \quad(\delta=10+3 \sqrt{11}) \\
F_{4}+F_{6}=11=X_{1}, \quad F_{6}+F_{13}=241=X_{2} \quad(\delta=11+2 \sqrt{30})
\end{gathered}
$$

and

$$
F_{5}+F_{21}=10951=X_{2} \quad(\delta=74+5 \sqrt{219}) .
$$

The above table contains only the information on $X_{\ell}=F_{n}+F_{m}$ with $n-m \geq 2$, but we can find the additional solutions when $n-m \leq 1$. Indeed, they are

$$
\begin{gathered}
F_{3}+F_{0}=2 F_{1}=2 F_{2}=2=X_{1}, \quad 2 F_{7}=26=X_{3} \quad(\delta=2+\sqrt{3}) \\
2 F_{5}=10=X_{1} \quad(\delta=10+3 \sqrt{11}) .
\end{gathered}
$$

For $\epsilon=-1$ :

$$
\begin{gathered}
F_{1}+F_{3}=3=X_{2}, \quad F_{3}+F_{5}=7=X_{3}, \quad(\delta=1+\sqrt{2}) \\
F_{12}+F_{23}=28801=X_{2} \quad(\delta=120+\sqrt{14401}) .
\end{gathered}
$$

Allowing for $m=0$ or $m \in\{n-1, n\}$, we get the additional solutions $F_{1}=F_{2}=1=X_{1}$ and $F_{4}=3=X_{2}$ when $\delta=1+\sqrt{2}$.

Note that in the cases $d \in\{219,14401\}$, we only found one value of $\ell$ such that $X_{\ell}$ has Zeckendorf representation with at most two terms (instead of two such $\ell$ ), which is why these $d$ are not included in the statement of the main result. 


\section{Acknowledgements}

C. A. G. was supported in part by Project 71079 (Universidad del Valle). F. L. was supported by grant CPRR160325161141 and an A-rated scientist award both from the NRF of South Africa and by grant no. 17-02804S of the Czech Granting Agency.

\section{References}

[1] H. Cohen, Number Theory. Volume I: Tools and Diophantine Equations, Springer, New York, 2007.

[2] J. H. E. Cohn, The Diophantine equation $x^{4}-D y^{2}=1$. II, Acta Arith. 78 (1997), 401-403.

[3] A. Dossavi-Yovo, F. Luca and A. Togbé, On the $x$-coordinates of Pell equations which are rep-digits, Publ. Math. Debrecen 88 (2016), 381-399.

[4] A. Dujella and A. Pethő, A Generalization of a Theorem of Baker and Davenport, Quart. J. Math. Oxford 49 (1998), 291-306.

[5] B. Faye and F. Luca, On x-coordinates of Pell equations which are repdigits, preprint, 2016.

[6] M. Laurent, M. Mignotte and Yu. Nesterenko, Formes linéaires en deux logarithmes et déterminants d'interpolation, J. Number Theory 55 (1995), 285-321.

[7] W. Ljunggren, Zur Theorie der Gleichung $X^{2}+1=D Y^{4}$, Avh. Norske Vid. Akad. Oslo I. Mat.-Naturv. 1942 (5), 27 pp.

[8] F. Luca, A. Montejano, L. Szalay and A. Togbé, On the $x$-coordinates of Pell equations which are Tribonacci numbers, Acta Arith., to appear.

[9] F. Luca and A. Togbé, On the x-coordinates of Pell equations which are Fibonacci numbers, Math. Scand., to appear.

[10] E. M. Matveev, An Explicit Lower Bound for a Homogeneous Rational Linear Form in the Logarithms of Algebraic Numbers, Izv. Math. 64 (2000), 1217-1269.

[11] W. R. Spickerman, Binet's formula for the Tribonacci numbers, The Fibonacci Quarterly 20 (1982), 118-120.

[12] Sun Qi, Yuan Sun Qi and Yuan Ping-Zhi, On the Diophantine equation $x^{4}-D y^{2}=1$, Advances in Math. (China) 25(1) (1996).

[13] Sun Qi and Yuan Ping-Zhi, A note on the Diophantine equation $x^{4}-D y^{2}=1$, Sichuan Daxue Xuebao, 34 (1997), 265-268.

[14] E. Zeckendorf. Représentation des nombres naturels par une somme de nombres de Fibonacci ou de nombres de Lucas, Bulletin de la Société Royale des Sciences de Liège 41 (1972), 179182. 